\title{
Ein Jahr DSGVO in Schulen
}

\author{
Hennig Dehnert/Annika Glahe/Sina-Marie Kunze/Ulrike Schulze
}

\section{Einleitung ${ }^{1}$}

Kaum ein anderes Thema wurde im letzten Jahr im schulischen Bereich so intensiv diskutiert wie die Datenschutzgrundverordnung (DSGVO). Der bisher in den Schulen eher als Randthema behandelte Datenschutz rückte durch die DSGVO und die große mediale Aufmerksamkeit dafür in den Fokus der Aufmerksamkeit. Nunmehr wird die DSGVO seit einem Jahr angewendet. Der Bundesbeauftragte für den Datenschutz und die Informationsfreiheit sieht in einem Jahr DSGVO „,einen Erfolg mit weiterem Steigerungspotential“. Die DSGVO habe den Datenschutz nicht nur in Deutschland und Europa, sondern sogar weltweit erheblich nach vorne gebracht. Viele Menschen und Unternehmen, die sich vorher nicht um den Datenschutz gekümmert haben, hätten sich mit dem Thema auseinandergesetzt. ${ }^{2}$ Der ehemalige Bundesbeauftragte für Datenschutz und Informationsfreiheit Peter Schaar sieht in einem Jahr DSGVO einen Grund zum Feiern. ${ }^{3}$ Dieser Beitrag möchte auf ein Jahr Anwendung der DSGVO in der schulischen Praxis in Niedersachsen zurückblicken, und untersuchen, ob in diesem Bereich ebenfalls ein solches positives Fazit gezogen werden kann.

\section{Die Bedeutung des Themas Datenschutz im Schulalltag}

Zentrale Zielsetzung des Datenschutzes ist der Schutz des allgemeinen Persönlichkeitsrechtes in Gestalt des Grundrechtes auf informationelle Selbstbestimmung. ${ }^{4}$ Dieses Grundrecht wurde erstmals im Volkszählungsurteil des Bundesverfassungsgerichtes anerkannt. ${ }^{5}$ Einschränkungen dieses Rechts auf „,informationelle Selbstbestimmung“ sind nur im überwiegenden Allgemeininteresse zulässig. Sie bedürfen einer verfassungsgemäßen gesetzlichen Grundlage, die dem rechtsstaatlichen Gebot der Normenklarheit entsprechen muss. ${ }^{6}$ Dieser Grundrechtsschutz muss auch in öffentlichen Schulen verwirklicht werden.

1 Die Autorinnen und der Autor sind bei der Niedersächsischen Landeschulbehörde tätig. Der Beitrag ist nicht in dienstlicher Eigenschaft verfasst und gibt ausschließlich die persönliche Auffassung der Autorinnen und des Autors wieder.

2 www.bfdi.bund.de/DE/Infothek/Pressemitteilungen/2019/17_EinJahrDSGVO.html (12.6.2019).

3 www.heise.de/newsticker/meldung/Kommentar-Ein-Jahr-DSGVO-ein-Grund-zum-Feiern-4433915.html (12.6.2019).

4 Schantz, P. in: Schantz/Wolff, Das neue Datenschutzrecht, 1. Auflage 2017, Rn. 136; Leeb, C.-M./Liebhaber, J., Grundlagen des Datenschutzrechts, JUS 2018, S. 534.

5 BVerfG, Urteil v. 15.12.1983, Az. 1 BvR 209, 269, 362, 420, 440, 484/83; ausführlich hierzu aus aktueller Sicht Simitis, S./Hornung, G./Spiecker, I. gen. Döhmann, in: Simitis/Hornung/Spiecker gen. Döhmann, Datenschutzrecht, Einleitung Rn. 28-48.

6 So wörtlich das BVerfG im 2. Leitsatz des Urteils v. 15.12.1983, Az. 1 BvR 209, 269, 362, 420, 440, $484 / 83$. 
Obwohl Schulen verglichen mit vielen Unternehmen und anderen öffentlichen Stellen kleine Organisationseinheiten sind, darf die Komplexität des Themas Datenschutz in der Schule nicht unterschätzt werden. Datenschutz bzw. die DSGVO wird im Schulalltag in vielen Situationen relevant. Schulen verarbeiten jeden Tag eine Vielzahl personenbezogener Daten ihrer Schülerinnen und Schüler. Über eine langjährige Schullaufbahn sammelt sich eine sehr große Menge personenbezogener Daten an. Auch personenbezogene Daten von Erziehungsberechtigten und Lehrkräften werden verarbeitet. So gut wie alle Schulen betreiben heute eine eigene Homepage. Schulen sind daher seit vielen Jahren im Alltag mit einer Vielzahl datenschutzrechtlicher Fragestellungen konfrontiert. Seit den 1970er Jahren erließen die Länder Datenschutzgesetze, die auch von den öffentlichen Schulen zu beachten waren. In Niedersachsen hat der Gesetzgeber in $\S 31 \mathrm{NSchG}$ eine generalklauselartige Erlaubnisnorm zur Verarbeitung personenbezogener Daten durch Schulen geschaffen. Schulen dürfen personenbezogene Daten der Schülerinnen und Schüler und ihrer Erziehungsberechtigten verarbeiten, soweit dies zur Erfüllung des Bildungsauftrags der Schule oder der Fürsorgeaufgaben, zur Erziehung oder Förderung der Schülerinnen und Schüler oder zur Erforschung oder Entwicklung der Schulqualität erforderlich ist. In den Schulen wurde pragmatisch mit dieser Regelung umgegangen. Aufregung verursachte das Thema Datenschutz kaum.

\section{Der anfängliche Umgang der Schulen mit der DSGVO}

Dies änderte sich im Frühjahr 2018 grundlegend. Je näher der 25.05.2018 - der Tag, ab dem die Regelungen der DSGVO angewendet werden mussten - rückte, desto deutlicher war in der Kommunikation mit den Schulen eine starke Verunsicherung wahrzunehmen. Bei vielen Schulen war eine massive Angst vor unbekannten neuen Regelungen, einhergehend mit der Befürchtung der Schulen, diese ohne juristische und technische Kenntnisse nicht umsetzen zu können, wahrzunehmen. Vermehrt äußerten die Schulen zudem eine immense Angst vor Haftung, insbesondere vor einer möglichen Verhängung von Bußgeldern gem. Art. 83 DSGVO. Diese Angst führte bei einigen Schulen zu Radikallösungen, wie beispielsweise das vollständige Deaktivieren der Schulhomepages, welche für gewöhnlich zum Zwecke der Außendarstellung mit Bildern von Schülerinnen und Schülern und Lehrkräften versehen sind. Auch erklärten einige Schulleitungen ihre Schulen auf Grund der DSGVO zur „fotofreien Schule“. Nicht wenige Schulen waren der Ansicht, dass die Umsetzung der Vorgaben der DSGVO für sie eine unüberwindbare Hürde darstelle.

\section{4 Überblick über Auswirkungen DSGVO für Schulen}

Vordringlichstes Ziel der DSGVO ist es, das Datenschutzrecht innerhalb Europas stärker zu vereinheitlichen und dadurch die Grundrechte und Grundfreiheiten und insbesondere ihr Recht auf Schutz personenbezogener Daten zu stärken. ${ }^{7}$ Um diese Zielsetzung zu erreichen, verfolgt die DSGVO einen ,one size fits it all“ Ansatz. ${ }^{8}$ Grundsätzlich gelten für alle Datenverarbeiter dieselben Vorschriften. Die kleine Grundschule auf dem Land mit nur sechs Lehrkräften hat

7 Vgl. Erwägungsgrund 2 der DSGVO.

8 Kritisch hierzu: Schulz, S., in: Gola, VO (EU) 2016/697, 2. Auflage 2018, Art. 6 Rn. 5; Veil, W., Die Datenschutz-Grundverordnung: des Kaisers neue Kleider, NVwZ 2018, S. 686 (693). 
daher prinzipiell dieselben datenschutzrechtlichen Anforderungen wie ein weltweit agierendes IT-Unternehmen mit tausenden Beschäftigten zu erfüllen. Die DSGVO normiert ein Verbot mit Erlaubnisvorbehalt. Gem. Art. 6 Abs. 1 S. 1 DSGVO ist die Verarbeitung personenbezogener Daten nur dann rechtmäßig, wenn einer der in Art. 6 Abs. 1 DSGVO normierten Erlaubnistatbestände erfüllt ist. ${ }^{9}$

Die Regeln der DSGVO führen für die Schulen zu neuen Verpflichtungen und Anforderungen. Dies sind z.B. die Erfüllung von Informations- und Auskunftspflichten gem. Art. $13 \mathrm{ff}$. DSGVO oder die Verpflichtung zur Meldung von Datenschutzverletzungen gem. Art. 33 DSGVO. Geht einer Schule beispielsweise einmal ein Klassenbuch verloren und ist dieses mitsamt der darin vermerkten personenbezogenen Daten nicht wieder auffindbar, stellt dies eine meldepflichtige Datenschutzverletzung dar. Die Schule muss in so einem Fall unverzüglich und möglichst binnen 72 Stunden die Datenschutzverletzung bei der Aufsichtsbehörde über eine dafür bereitgestellte Plattform anzeigen. Als besonders belastend werden von den Schulen die Dokumentationspflichten wahrgenommen. Zentraler Baustein bei der Erfüllung der Dokumentationspflichten ist die Führung eines Verzeichnisses von Verarbeitungstätigkeiten gem. Art. 30 DSGVO. In dieses Verzeichnis sind sämtliche ganz oder teilweise automatisierte Verarbeitungen, also digitale Datenverarbeitungsprozesse, einzutragen. Ebenfalls darin aufzunehmen sind analoge, nichtautomatisierte Verarbeitungen personenbezogener Daten, die in einem Dateisystem gespeichert sind oder gespeichert werden sollen. Jeder dieser Datenverarbeitungsprozesse ist im Verzeichnis von Verarbeitungstätigkeiten zu beschreiben und zu dokumentieren. Neben der Führung des Verzeichnisses von Verarbeitungstätigkeiten haben die Schulen weitere Dokumentationspflichten zu erfüllen. Das sind vor allem die Dokumentation der Ergebnisse von Datenschutzfolgeabschätzungen gem. Art. 35 DSGVO und das Vorliegen von Einwilligungen gem. Art. 7 DSGVO.

Die Sensibilität für Datenschutz ist mittlerweile jedoch nicht nur bei den Schulen, sondern auch bei Erziehungsberechtigten und (ehemaligen) Schülerinnen und Schülern festzustellen. So haben sich Schulen wesentlich häufiger als früher mit Akteneinsichtsgesuchen von Betroffenen zu befassen. Ersucht ein Betroffener um Auskunft über die bei der Schule gespeicherten personenbezogenen Daten nach Art. 15 DSGVO, muss die Schule die Informationen unverzüglich, in jedem Fall aber innerhalb eines Monats nach Eingang des Antrags zur Verfügung stellen, vgl. Art. 12 DSGVO. Kommt eine Schule diesem Ersuchen nicht fristgerecht nach, ist es durchaus möglich - und auch bereits vorgekommen -, dass der Ersuchende von seinem Beschwerderecht bei der Datenschutzaufsichtsbehörde gem. Art. 77 DSGVO Gebrauch macht und die Schule zu einer fristgebundenen Stellungnahme aufgefordert wird. Die Befürchtung der öffentlichen Schulen, ihnen drohe die Verhängung von Bußgeldern ist nicht zutreffend, denn $\S 20$ Abs. 5 des neugefassten Niedersächsischen Datenschutzgesetzes gibt der von der oder dem Landesbeauftragten geleiteten Behörde nur dann die Befugnis, gegenüber öffentlichen Stellen Geldbußen zu verhängen, soweit diese als Unternehmen am Wettbewerb teilnehmen. ${ }^{10}$ Das ist bei öffentlichen Schulen nicht der Fall.

9 Buchner, B./Petri, T., in: Kühling/Buchner, Datenschutz-Grundverordnung/BDSG, 2. Auflage 2018, Art. 6 Rn. 13; Heberlein, H., in: Ehmann/Selmayr, DS-GVO, 2. Auflage 2018, Art. 6 Rn. 1.

10 In anderen Ländern gibt es vergleichbare Regelungen: Z.B. § 32 des Datenschutzgesetzes NordrheinWestfalen (DSG NRW) vom 17. Mai 2018; Art. 22 Bayerisches Datenschutzgesetz (BayDSG) vom 15. Mai 2018. 


\section{Unterstützung der Schulen durch die Schulaufsichtsbehörde bei der Vorberei- tung auf die DSGVO}

Bei der Vorbereitung der öffentlichen Schulen in Niedersachsen auf die Umsetzung der DSGVO stellte die Niedersächsische Landesschulbehörde als zuständige Schulaufsichtsbehörde den Servicegedanken in den Mittelpunkt. Der Fokus lag nicht auf der Aufsicht, ob die Schulen ausreichende Vorbereitungen für die Umsetzung der DSGVO treffen, sondern auf der Beratung und der Unterstützung der Schulen. Dieser Ansatz ist angesichts der enormen Anzahl von öffentlichen Schulen in Niedersachsen, ${ }^{11}$ die alle als Verantwortliche im Sinne von Art. 4 Nr. 7 DSGVO unabhängig voneinander die Anforderungen der DSGVO zu erfüllen haben, enorm herausfordernd.

Um diese Herausforderung bewältigen zu können, erfolgte ein mehrstufiges Vorgehen. Zielsetzung der ersten Stufe war es, den Schulen eine Übersicht über die bei der Umsetzung der DSGVO anstehenden Aufgaben zu geben. Dadurch sollten den Schulen die Verunsicherung und die Ängste genommen werden. Um dieses Ziel zu erreichen, stellten die Autorinnen und der Autor dieses Beitrages die DSGVO in Dienstbesprechungen der Schulleitungen vor und referierten über die umzusetzenden Maßnahmen sowie die bereits bestehenden und geplanten umfangreichen Unterstützungsleistungen der Niedersächsischen Landesschulbehörde. Zeitgleich wurde ein umfangreiches Onlineangebot geschaffen. So lassen sich auf der Homepage der Niedersächsischen Landesschulbehörde zahlreiche Informationstexte und Vorlagen finden. Dieser Prozess konnte im Herbst 2018 abgeschlossen werden. Alle Schulen in Niedersachsen wurden in die Lage versetzt, sich umfassend über die Anforderungen durch die DSGVO zu informieren.

In einer 2. Stufe erhielten die Schulen Unterstützung durch landesweit stattfindende vertiefende Informationsveranstaltungen und weitere Materialien, z.B. Mustereinträge für das Verzeichnis für Verarbeitungstätigkeiten. Diese Unterstützungsleistungen werden nach wie vor angeboten und von den Schulen auch nachgefragt. Bei darüber hinaus gehenden speziellen Fragestellungen, insbesondere solche, die sich aus der Digitalisierung des Unterrichtes, die durch Mittel des Digitalpaktes enorm gefördert und beschleunigt werden wird, werden die Schulen ebenfalls von der Niedersächsischen Landesschulbehörde unterstützt. Diese 3. Stufe des Beratungs- und Unterstützungsprozesses hat bereits begonnen und wird in den nächsten Monaten angesichts der bevorstehenden Förderung des mobilen, digitalen Lernens durch den Digitalpakt Schule immer mehr Bedeutung erlangen. Durch den Digitalpakt Schule ${ }^{12}$ und der damit verbundenen Geldausschüttung von insgesamt fünf Milliarden EURO zur Förderung der Digitalisierung in Schulen liegt es auf der Hand, dass mobiles Lernen in Tabletklassen verstärkt Einzug in die Schulen finden wird. Die Schulen müssen bei der Einführung von Tablets im Unterricht wesentliche (datenschutz-) rechtliche Aspekte beachten. Bei der Umsetzung dieser Anforderungen besteht die zusätzliche Herausforderung, dass die Schulen über keine landeseinheitliche IT-Ausstattung verfügen. In Niedersachsen ist nämlich die sächliche Ausstattung der Schulen wie auch in anderen Bundesländern keine Landesaufgabe, sondern Aufgabe der kommuna-

11 Im Moment gibt es ca. 2800 öffentliche Schulen in Niederachsen. Aktuelle Informationen zur Anzahl der Schulen in Niedersachsen sind hier abrufbar: www.landesschulbehoerde-niedersachsen.de/organisati on (12.6.2019).

12 Hintergrundinformationen sind auf der Homepage des Bundesministeriums für Bildung und Forschung abrufbar: www.bmbf.de/de/wissenswertes-zum-digitalpakt-schule-6496.php.(12.6.2019). 
len Schulträger. ${ }^{13}$ Hinzu kommt, dass die in Schulen tätigen Lehrkräfte in der Regel weder ein Studium der Rechtswissenschaften absolviert noch vertiefte technische Kenntnisse haben. Vor diesem Hintergrund erschließt sich ohne Weiteres, dass eine Vielzahl von Schulen die Anforderungen der DSGVO in der Praxis ohne externe Unterstützung nicht leisten können.

Aus dem Vorstehenden wird deutlich, dass den öffentlichen Schulen in Niedersachsen in Bezug auf die Umsetzung der DSGVO bereits umfangreiche Beratungs- und Unterstützungsangebote zur Verfügung stehen. Diese wurden und werden auch zahlreich genutzt. Dank dieser standardisierten Beratungs- und Unterstützungsangebote konnte den Schulen die anfängliche Angst und Verunsicherung genommen werden. Die Schulen wurden in die Lage versetzt, die grundlegenden Anforderungen der DSGVO mit vertretbarem Aufwand zu erfüllen. Diese Strategie, die Schulen durch zentral bereitgehaltene Informationen und Musterdokumente zu unterstützen, hat allerdings auch ihre Grenzen. Sofern in den Schulen vor Ort keine Personen verfügbar sind, die über datenschutzrechtliche Grundkenntnisse verfügen, muss sich die Umsetzung der Datenschutzverordnung in vielen Fällen auf das Abarbeiten von Checklisten und das Ausfüllen von Musterdokumenten beschränken. Eine vertiefte, selbständige Auseinandersetzung mit den Anforderungen der DSGVO und das selbständige Finden individueller Lösungen kann von vielen Schulen nicht geleistet werden. Fest steht daher, dass bei vielen Schulen in Sachen DSGVO nach wie vor ein hoher individueller Beratungsbedarf besteht und wahrscheinlich auch bestehen bleiben wird.

\section{Versuch einer Bewertung der Auswirkungen der DSGVO auf die Schulen}

Nunmehr soll der Blick auf die Fragestellung gerichtet werden, ob die DSGVO nach einem Jahr praktischer Anwendung zu einer Stärkung des Schutzes personenbezogener Daten geführt hat. Uneingeschränkt positiv hervorzuheben ist, dass die intensive Debatte über das Thema DSGVO das Thema Datenschutz in den Schulen auf die Tagesordnung gebracht hat. Nachdem die Schulen die anfängliche Furcht vor der DSGVO überwunden hatten, haben sich die Schulen des Themas Datenschutz angenommen und den Weg zur Umsetzung der DSGVO gemacht. Dabei ist es den meisten Schulen gelungen, die grundlegenden Anforderungen, wie z.B. die Erfüllung der Informationspflichten, sicherzustellen. Dies hat zu einer gewissen Verbesserung des Datenschutzniveaus in den Schulen geführt. Kaum eine Schule veröffentlicht heute noch Bilder von Schülerinnen und Schülern auf ihrer Homepage ohne zuvor Einwilligungen von den Betroffenen einzuholen. Die gewissenhafte Erfüllung dieser Aufgaben geht mit einer entsprechenden Zeitinvestition einher. Viele Schulen investieren sehr viel Arbeit und Zeit in die Erfüllung der datenschutzrechtlichen Anforderungen und erreichen dadurch eine Verbesserung des Datenschutzniveaus. Dennoch gibt es Bereiche, in denen die Schulen mit den ihnen zur Verfügung stehenden zeitlichen und personellen Ressourcen nachvollziehbar an ihre Grenzen stoßen. Exemplarisch sind hier die Themen die Erfüllung der Dokumentationspflichten und die Datenschutzfolgeabschätzungen zu nennen.

Insgesamt fällt die Bilanz gemischt aus: An vielen Schulen hat sich das Datenschutzniveau spürbar verbessert. Ein neues positives Datenschutzbewusstsein, in dem Sinne, dass im Datenschutz mehr als das Abarbeiten formaler Anforderungen gesehen wird, hat sich trotz aller Beratung und Unterstützung zwar an vielen, aber noch nicht an allen Schulen einstellen können.

13 Siehe $\S 113$ NSchG. 
Dies ist zu bedauern, aber angesichts der Vielzahl der Dokumentationspflichten durchaus nachvollziehbar. Bei der bevorstehenden Evaluation der DSGVO ${ }^{14}$ sollte dies in den Blick genommen werden. Die Zukunft wird zeigen, ob der „one size fits it all“ der DSGVO Ansatz im Schulbereich weiterhin uneingeschränkt gelten wird.

\section{Fazit}

Den Schulen konnte die anfängliche Angst vor der DSGVO genommen werden. Fakt ist, dass in Schulen weiter das Bewusstsein für Datenschutz implementiert werden muss und die Schulen - insbesondere im Hinblick auf die zunehmende Digitalisierung des Unterrichtes - auch zukünftig verstärkt mit datenschutzrechtlichen Regelungen konfrontiert sein werden.

Fakt ist aber auch, dass die Schulen in Niedersachsen bei allen datenschutzrechtlichen Fragen von der Niedersächsischen Landesschulbehörde unterstützt werden und auch weiterhin kein Grund besteht, die DSGVO als unüberwindbare Hürde anzusehen.

Dr. Henning Dehnert, Niedersächsische Landesschulbehörde, Regionalabteilung Osnabrück, Mühleneschweg 8, 49090 Osnabrück, Tel: 0541 77046-417, Fax: 0541-77046-8-103, henning.dehnert@nlschb.niedersachsen.de

Annika Glahe, Niedersächsische Landesschulbehörde, Regionalabteilung Hannover, Mailänder Str. 2, 30539 Hannover, Tel: 0511 106-2443, Fax: 0511 106-992853, annika.glahe@nlschb.niedersachsen.de

Sina-Marie Kunze, Niedersächsische Landesschulbehörde, Regionalabteilung Braunschweig, Wilhelmstr. 62 - 69, 38100 Braunschweig, Tel: 0531 484-3429, Fax: 0531 484-3483, sina-marie.kunze@nlschb.niedersachsen.de

Ulrike Schulze, Niedersächsische Landesschulbehörde, Regionalabteilung Lüneburg, Auf der Hude 2, 21339 Lüneburg, Tel: 04131 15-2320, Fax: 04131 15-2930, ulrike.schulze@nlschb.niedersachsen.de

14 Art. 97 Abs. 1 S. 1 DSGVO sieht vor, dass die Kommission dem Europäischen Parlament und dem Rat einen Bericht über die Bewertung und Überprüfung der DSGVO bis zum 25. Mai 2020 und danach alle vier Jahre vorlegt. 